quarter of 2001-02. ${ }^{2}$ The estimated annual use fell from about 66000 to 51000 treatments; if this decline in ECT use continued at the same rate, then the straight line extrapolated from the last data point would reach zero by the year 2011-12. There has never been another national survey. A partial survey of English ECT clinics in the first quarter of 2006 suggested a further fall, to only about 27000 , which was in line with the extrapolation. ${ }^{3}$ The re-appraisal prompted us to review the rate of ECT usage in our clinic since 2006.

The rates of ECT usage in 2006 and 2011 were almost identical, that is, 0.82 and 0.83 individual treated patients per 10000 population in the City of Edinburgh. Likewise, the rates in the intervening years were also almost identical. We therefore conclude, at least for Edinburgh, that the rate of ECT use has been stable for the past 6 years.

The electronic data collection system in our ECT clinic was updated at the end of 2004, and included a record of the primary psychiatric diagnosis of referred patients. The number of referred patients diagnosed with a severe depressive episode (both with and without psychotic features) varied little in these 6 years, from 23 to 28 patients. This gave a crude referral rate of 25 patients with severe depression per year per total population of 500000 .

If we are treating just as many patients with severe depression as 5 or 6 years ago, then this must continue to be resourced. It is not just ECT practitioners that have heard the suggestion about the demise of ECT. Senior managers locally have expressed surprise to hear that there is still a need for the ECT clinic at the Royal Edinburgh Hospital. This concerned us because when the availability of ECT was reduced in Glasgow, ECT use fell. ${ }^{4}$ The search for the Holy Grail is laudable, but patients with severe depression still need access to ECT.

1 Allan C, Kalu U-G, Sexton CE, Ebmeier KE. Transcranial stimulation in depression. Br J Psychiatry 2012; 200: 10-11.

2 Department of Health. Electro Convulsive Therapy: Survey Covering the Period from January 2002 to March 2002, England. Statistical Bulletin 2003/ 08. Department of Health, 2003.

3 Bickerton D, Worrall A, Chaplain R. Trends in the administration of electroconvulsive therapy in England. Psychiatr Bull 2009; 33: 61-3.

4 McAllister M, Crabb J, Brodie D, Krishandas R. Effect of closing facilities on electroconvulsive therapy use in Glasgow. J ECT 2011; 27: 131-3.

Allan Scott, Royal Edinburgh Hospital, Morningside Terrace, Edinburgh EH10 5HF, UK. Email: Fiona.J.Morrison@nhslothian.scot.nhs.uk; Simone Burns, Royal Edinburgh Hospital, Edinburgh, UK

doi: $10.1192 / b j p .200 .5 .428 b$

\section{Methodological discrepancies in the update of a meta-analysis}

Leichsenring \& Rabung ${ }^{1}$ reported that long-term psychodynamic psychotherapy (LTPP) is superior to less intensive forms of psychotherapy in complex mental disorders. Based on 10 trials, they found an overall effect size (ES) of 0.55 . We found several methodological discrepancies in their study.

First, it seems surprising that the Q-test indicated no significant unexplained variance, as the between-group effect size of one of the primary studies ${ }^{2}(\mathrm{ES}=1.76)$ is quite outstanding in Fig. 2. To shed light on this issue, we recalculated the overall effect size using a random effects meta-analysis based on the values from Fig. 2. Our meta-analysis replicated Leichsenring \& Rabung $^{1}$ in the main. In contrast to Leichsenring \& Rabung however, we found a significant unexplained variance $(Q=25.33$, d.f. $=9$, $P=0.003)$ and a larger overall confidence interval of $0.29-0.82$ (in contrast to $0.41-0.67$ as reported by Leichsenring \& Rabung). Additionally, computing an outlier analysis, a significant outlying study effect size was found $(P<0.001)$. Including the moderator considering the impact of this study yields an effect size of 0.44 (95\% CI 0.27-0.61, $P<0.001$ ). The moderator effect, interpreted as the difference between the effect of the outlying study and the grand mean, was 1.32 (95\% CI $0.57-2.07, P<0.001)$. After removing the outlying study, there was no significant unexplained variance $(Q=11.56$, d.f. $=8, P=0.172)$.

Second, we calculated the fail-safe $N$ according to Rosenthal; ${ }^{3}$ 16 non-published studies with an effect size of 0 had to be included in the analysis to change the results of the meta-analysis $(\mathrm{ES}=0.44)$ from significant to non-significant $(\mathrm{ES}<0.16)$. As 16 is below $55(5 K+10)$, the effect cannot be regarded as robust.

Last, to gain better insight into the interpretation of the overall effect size as small, medium or large, we calculated a Bayesian meta-analysis following Higgins et al's methodology. ${ }^{4}$ The Bayesian analysis essentially replicated the findings of our random effects meta-analysis. In addition, we found the probability of the overall effect size to be small $(\mathrm{ES}<0.5)$ at $72.5 \%$. Thus, in contrast to Leichsenring \& Rabung, ${ }^{1}$ we found that the overall effect size was small rather than medium or large.

Therefore, we would greatly appreciate caution against a conclusion that the overall effectiveness of LTPP for treating complex mental disorders should now be considered as definitely proven.

1 Leichsenring F, Rabung S. Long-term psychodynamic psychotherapy in complex mental disorders: update of a meta-analysis. Br J Psychiatry 2011; 199: $15-22$

2 Bateman A, Fonagy P. The effectiveness of partial hospitalization in the treatment of borderline personality disorder, a randomized controlled trial. Am J Psychiatry 1999; 156: 1563-9.

3 Rosenthal R. The file drawer problem and tolerance for null results. Psychol Bull 1979; 86: 638-41.

4 Higgins JPT, Thompson SG, Spiegelhalter DJ. A re-evaluation of randomeffects meta-analysis. J $R$ Statist Soc A 2009; 172: 137-59.

Soeren Kliem, PhD student, Department of Psychology, Technical University of Braunschweig, Germany. Email: soerenkliem@gmx.net; Johannes Beller, Christoph Kroeger, Department of Psychology, Technical University of Braunschweig, Germany

doi: $10.1192 /$ bjp.200.5.429

Author's reply: When trying to replicate some results of our meta-analysis, ${ }^{1}$ Kliem and colleagues reported some methodological discrepancies. ${ }^{2}$ These discrepancies, however, are due to modifications in their statistical approach as compared with the one we originally reported.

First, in contrast to our results, ${ }^{1}$ Kliem et al reported significant heterogeneity between studies for overall outcome as indicated by the $Q$ statistic. As stated in our meta-analysis, we had aggregated the effect size estimates across studies, adopting a random effects model, which is more appropriate than a fixed effects model if the aim is to make inferences beyond the observed sample of studies. ${ }^{1,2}$ Applying a random effects model, the aggregated effect size for overall outcome was 0.54 , and heterogeneity was not significant $\left(Q=11.72, P=0.23, I^{2}=23\right)$. Thus, there was no need for an additional outlier analyses or for the exclusion of any study. As Rosenthal's fail-safe $N$ was 66, which is above $60(5 K+10)$, the effect can be regarded as robust. Kliem et al, however, apparently applied the fixed effects model to test for heterogeneity. The use of a fixed effects model, however, addresses another research question and consequently yields different results. 